\title{
Adjacency Matrix
}

National Cancer Institute

\section{Source}

National Cancer Institute. Adjacency Matrix. NCI Thesaurus. Code C63897.

A matrix with rows and columns labeled by graph vertices, with a 1 or 0 in position (vi,vj) according to whether vi and vj are adjacent or not. 